\title{
The Association between Nutritional Parameters Used in Nutritional Risk Assessment (NRA) Tools in Hospitalized Malnourished COVID-19 Patients: A Systematic Review
}

\author{
Sanambar Sadighi ${ }^{1}$ (D), Sana Mohseni ${ }^{1}$ (D), Sayyideh Forough Hosseini ${ }^{1}$ (D), Orkideh Olang ${ }^{1}$ (D), Seyedeh
} Mohaddeseh Khatami ${ }^{1}$ (D) Maryam Rezaii Salim ${ }^{1}$ (D) Johane Allard ${ }^{2}$ (D) and Mohammad Ali Shafiee ${ }^{1^{*}}$ (iD)

${ }^{1}$ Division of General Internal Medicine, Department of Medicine, Toronto General Hospital, Canada

${ }^{2}$ Department of Medicine, University of Toronto, Toronto, Canada

\begin{abstract}
Objective: With the increasing COVID-19 pandemic healthcare burden, preventive and modifying measures could significantly affect patients' outcome. In this regard, nutritional assessment tools, primarily based on laboratory inflammatory markers, could be affected by cytokine release, and thereby may not accurately reflect nutritional status of the severely ill patients. This study aimed to evaluate the relationship of those factors with malnutrition in COVID-19 patients.

Methods: We performed a systematic literature search, using various databases, including Medline, Embase, APA PsycINFO, Cochrane Database of Systematic Reviews via Ovid, and CINAHL via Ebsco. PRISMA guidelines used for study selection and data extraction.

Result: Sixteen descriptive studies out of 3,117 articles with 4,155 participants included. The frequency of malnutrition in reviewed studies ranged from $17 \%$ to $92 \%$. Lower serum albumin was associated with more severe malnutrition in Nutritional Risk Assessment (NRA). Although there was a clear consensus on serum albumin association with malnutrition, there were more controversial results for blood lymphocyte count and other inflammatory indices. Four out of eleven studies that used anthropometric nutritional assessment tools showed body mass index (BMI) significantly correlated with malnutrition.

Conclusion: Whereas serum albumin generally is affected by the severe inflammatory nature of COVID-19 disease, this systematic review revealed a significant association between albumin level and malnutrition; this association was not observed with inflammatory biomarkers. The complex status of hospitalized COVID-19 patients needs further prospective studies to clarify the role of the body composition measures in nutritional decision making.
\end{abstract}

Keywords

COVID-19, Malnutrition, Nutritional risk screening, Inflammation, Anthropometry

\section{Introduction}

The coronavirus pandemic has created a huge health crisis with significant financial impacts and resource shortcomings. During this overwhelming situation, appropriate preventive, diagnostic, and therapeutic interventions are crucial. There are considerable nutritional concerns around COVID-19 disease and many new studies paid particular attention to malnutrition screening and micro-macro nutrient requirements among COVID-19 patients [1]. The prevalence of malnutrition reported in different studies varies according to many risk factors, including age, comorbidities, gastrointestinal symptoms of patients, and extended stay in intensive care units $[2,3]$.

There is a growing need for nutritional screening
*Corresponding author: Mohammad A Shafiee, MD, MSc, FRCPC, Division of General Internal Medicine, Department of Medicine, Toronto General Hospital, Toronto, ON M2G 2C4, Canada, Tel: 416-340-4800 ext 6244, Fax: 416-595-5826

Accepted: February 10, 2022

Published online: February 12, 2022

Citation: Sadighi S, Mohseni S, Hosseini SF, et al. (2022) The Association between Nutritional Parameters Used in Nutritional Risk Assessment (NRA) Tools in Hospitalized Malnourished COVID-19 Patients: A Systematic Review. J Healthcare 5(1):106116

Copyright: (c) 2022 Sadighi S. et al This is an open-access article distributed under the terms of the Creative Commons Attribution License, which permits unrestricted use, distribution, and reproduction in any medium, provided the original author and source are credited. 
Citation: Sadighi S, Mohseni S, Hosseini SF, et al. (2022) The Association between Nutritional Parameters Used in Nutritional Risk Assessment (NRA) Tools in Hospitalized Malnourished COVID-19 Patients: A Systematic Review. J Healthcare 5(1):106-116

tools to promptly assess COVID-19 patients vulnerable to malnutrition to reduce complications and provide the best suitable treatment options. Health care systems need to be encouraged to identify and utilize the proper nutrition indices. Many studies are related to different nutritional parameters used in Nutritional Risk Screening (NRS) tools and the validity of such measures in hospitalized patients with multiple medical conditions; however, their validity in patients with COVID-19 has not been thoroughly clarified [2].

Many Nutritional Risk Assessment (NRA) tools are based on anthropometric factors such as Body Mass Index (BMI) [4]. There is a controversy around the BMI issue in severe COVID cases. A mean BMI of $30 \mathrm{~kg} / \mathrm{m}^{2}$ and more was reported among COVID-19 inpatients as a common critical factor [1]. Other researchers revealed low $\mathrm{BMI}$ as a significant issue, especially in elderly-COVID-19 patients [5]. In addition to those extreme ranges in anthropometric measures, different inflammatory biomarkers are used in NRA of COVID-19 patients [3,6].

COVID-19 infection causes an excessive inflammatory response, influencing laboratory biomarkers, including albumin, total white blood cell count (WBC), lymphocyte count, lactate dehydrogenase (LDH), and C-reactive protein (CRP). Elevated markers of systemic inflammation, used in the laboratory-based NRAs such as prognostic nutritional index (PNI) and controlling nutritional status (CONUT), might overestimate the prevalence of malnutrition in COVID-19 patients. Albumin is required to synthesize the acute phase reactants, leading to lower levels of albumin during any infections including COVID-19 [7]. Decreasing serum albumin also could be related to inflammatory response, not the actual patients' nutritional state.

There is a gap between recent information with a wide range of discrepancies in the prevalence of malnutrition from $17 \%$ [8] to more than $92 \%$ [9] recorded in different studies. The nature of COVID-19 infection, which influences nutritional biomarkers and anthropometric measures. This systematic review aims to clarify whether there is an association between nutritional and inflammatory parameters used in screening tools for COVID-19 inpatients.

\section{Subjects and methods}

The systematic review protocol is submitted in the International prospective register of systematic reviews. This systematic review was conducted based on Preferred Reporting Items for Systematic Reviews and (PRISMA) guidelines.

\section{Search strategy}

A systematic literature search was conducted to investigate the relationship between nutritional and inflammatory parameters among malnourished COVID-19 patients diagnosed with different nutritional assessment tools, using Medline, Embase, APA PsycINFO, and Cochrane Database of Systematic Reviews via Ovid, CINAHL via Ebsco up to November 08, 2021. Relevant articles were captured applying Medline's MeSH terms (adjusted for the databases mentioned above), "COVID-19," "Nutrition assessment,"
"Malnutrition," "nutritional status," "Anthropometry," and related terms, inflammatory factors, including possible adjacencies.

\section{Inclusion and exclusion}

Observational studies (longitudinal, case-control, crosssectional, and case-series) which reported the clinical anthropometric measurements and or inflammatory markers in hospitalized COVID-19 adult patients (18-years-and-older) with different nutritional status were considered eligible for inclusion in the study.

Animal studies, studies with the study population of pregnant women, and pediatric patients were excluded. Of note, non-English articles, study protocols, review articles, conference abstracts were excluded as well. Articles with relevant abstracts which needed further investigation were considered for full-text review, and eligible studies were included for data extraction. Title and abstract screening, full text review, and data extraction were performed by two trained medical graduates independently (OO, MK), and disagreements were discussed with one of the peers (SFH).

\section{Study population}

Studies that reported the anthropometric measurements and inflammatory biomarkers in hospitalized malnourished COVID-19 adult patients (> 18 years) were included. COVID-19 diagnosis in the included studies was confirmed by positive results on real-time polymerase chain reaction assays (OneStep real-time PCR Kits) for SARS-CoV-2 from nasopharyngeal swab specimens.

\section{Measurements}

In the included studies, nutritional status was assessed with anthropometric and laboratory-based measures. Anthropometric measures consist of Nutritional Risk Screening 2002 (NRS-2002) [10,11], Mini Nutritional Assessment (MNA) [5], Subjective Global Assessment (SGA) [2], and Global Leadership Initiative on Malnutrition (GLIM) criteria based on body mass index BMI (BMI), weight loss, dietary intake decrease, severity of an acute illness, muscle mass, and neuropsychological problems [4]. Laboratory based measures use, serum albumin, serum cholesterol, and or lymphocyte count. They consist of Nutrition Risk Index (NRI) = $1.519 \times$ serum albumin, $\mathrm{g} / \mathrm{L})+0.417 \times($ present weight/usual weight $\times 100$ ] [12], Prognostic Nutrition Index $(P N I)=10 \times$ serum albumin $(\mathrm{g} / \mathrm{dL})+0.005 \times$ peripheral lymphocyte count $\left(/ \mathrm{mm}^{3}\right)[13]$ and Controlling Nutritional (CONUT) status $=10 \times$ the serum albumin value $(\mathrm{g} / \mathrm{dl})+0.005 \times$ the total lymphocyte count in peripheral blood $\left(\right.$ per $\mathrm{mm}^{3}$ ) [14].

Moreover, various inflammatory and nutritional biomarkers such as albumin, prealbumin, CRP, platelet, Interleukin 6 (IL-6), D-dimer, LDH, Ferritin and procalcitonin were reported in the selected studies.

\section{Study's outcome}

The association of biochemical and inflammatory biomarkers with malnourishment diagnosed with different 
nutritional assessment tools in COVID-19 patients was investigated as the primary outcome. Secondly, correlation of different nutritional assessment tools was studied. Which ones are more associated with the diagnosis of malnutrition in COVID-19 patients?

\section{Quality assessment of studies}

As the study designs of articles which were evaluated in this review were observational, we used Grading of Recommendations, Assessment, Development, and Evaluation (GRADE) Newcastle-Ottawa Scale to assess the risk of bias and quality of articles in the selection process [15].

\section{Results}

\section{Study characteristics}

Using comprehensive search strategies, we identified
3,221 studies. After title and abstract screening and excluding duplicates $(3,117), 104$ articles were selected for full-text review. Excluding irrelevant ones, we included 16 articles in this systematic review. Figure 1 shows the PRISMA flowchart for the article selection process.

\section{Quality assessment and risk of bias in included studies}

The Newcastle-Ottawa Quality Assessment Scale was used to assess the quality and risk of bias of included studies. Three categories of population selection, comparability, and outcome were evaluated by this tool. If three out of four criteria in the selection category, two out of three criteria in outcome category, and one out of two in comparability category were met the study was categorized as "good". If the number of met criteria in the selection category decreased to two with

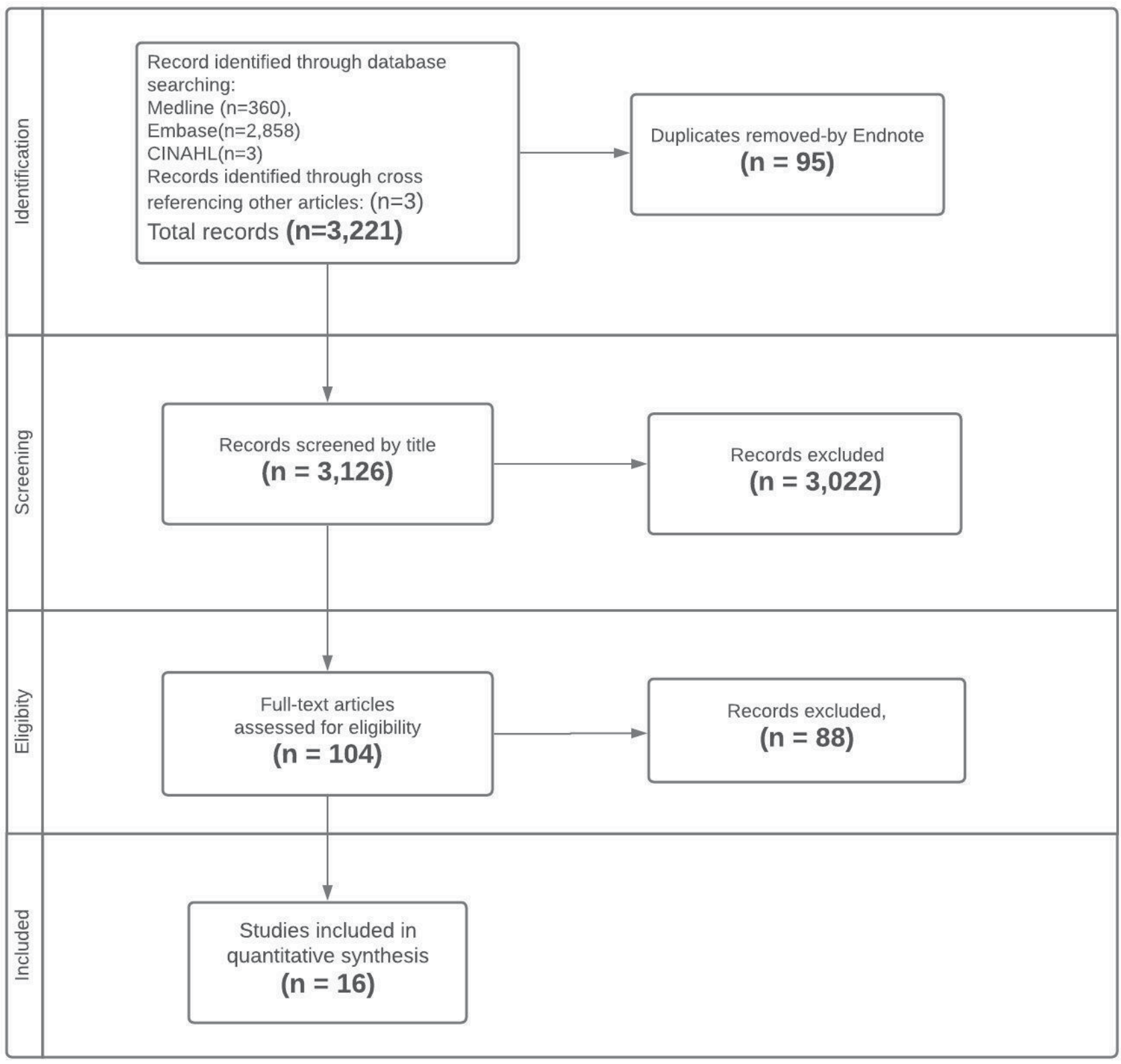

Figure 1: Flow chart of included articles for review. 
Citation: Sadighi S, Mohseni S, Hosseini SF, et al. (2022) The Association between Nutritional Parameters Used in Nutritional Risk Assessment (NRA) Tools in Hospitalized Malnourished COVID-19 Patients: A Systematic Review. J Healthcare 5(1):106-116

the same scoring in the other two categories, the quality of study would be "fair" [15] Thirteen articles had good quality. The mean score of articles in selection, comparability, and outcome categories were $2.8,1.3$ and 2.4 respectively. The patient groups in three studies were not a true representative of the population $[9,11,16]$. One study did not divide the population into malnourished and normal groups [3]. There was just one article with poor quality in which the definition of malnutrition was not followed according to the study's prior description; therefore, the groups could not be compared on the basis of the study [16].

\section{Results of the systematic review}

Sixteen descriptive studies with 4,155 participants were included. All patients were admitted to the hospital, and their diagnosis of COVID-19 was confirmed with RT-PCR. Four studies included only ICU patients $[10,11,13,16]$.

These studies categorized their included patients based on their nutritional status as normal (no malnutrition) ( $\mathrm{n}=$ 1,137 ), mild malnutrition ( $n=31$ ), moderate malnutrition ( $n$ $=912)$, severe malnutrition $(n=21)$, at risk of malnutrition ( $n=899$ ), and 705 patients as malnourished without further details. There was one study with 450 participants which did not divide patients based on their nutritional status. However, as the researchers provided the data related to different inflammatory markers and biochemical nutritional measures, that study was included [3]. The percentage of malnourished patients in four studies was $85 \%$ or more $[9,11,12,16]$ and in one it was $17 \%$ (Table 1) [8].

Eleven studies used nutritional screening tools which were based on clinical anthropometric findings. Six of them used NRS-2002 [9-12,16,17]. Two used SGA [2,8] and another two used GLIM and categorized patients by using clinical parameters [18,19]. Another one used the MNA-sf, a validated questionnaire for patients older than 65 [5]. One of the articles compared Bioelectrical Impedance Analysis with NRS2002 as well [9]. The remaining studies used measures based on some laboratory factors for dividing patients into different nutritional state categories. Three studies used PNI $[3,13,20]$. One used CONUT [6] and one used serum prealbumin as the nutritional state screening tool [21].

\section{Studies used clinical anthropometric measures to examine nutritional status}

Nutritional status in three studies was examined with NRS-2002 among ICU patients $[10,11,16]$. Czaplie and Kupeli studies did not find a significant correlation between NRS score with blood lymphocyte count or with serum albumin $[11,16]$. The NRS score of participants in the Kupeli study was $5.9 \pm 1.1$. Furthermore, all patients had low hemoglobin $(11.9 \pm 2.4 \mathrm{~g} / \mathrm{dL})$, and serum albumin $(29.7 \pm 5.3 \mathrm{~g} / \mathrm{L})[16]$. Frequency of malnutrition in Alikiaii study was $41.5 \%$ (Score $\geq$ 5 ) and they found a significant correlation of NRS with serum albumin $(-0.23 ; p=0.05)$. Mean serum albumin in those whose score was equal to or higher than five was significantly lower than those with score three ( $3 \pm 0.5$ vs. $3.2 \pm 0.6 \mathrm{~g} / \mathrm{dl}$ ). However, they did not confirm any significant correlation of NRS score with LDH, CRP and CPK, and or AST [10].
There were three more studies that used NRS-2002 in hospitalized patients who were not in ICU $[9,12,17]$. Liu A, et al. found lower BMI in those with a higher NRS-2002 score $(p=0.029)$. Furthermore, they discovered that NRS score significantly correlated with serum albumin $(\mathrm{g} / \mathrm{L})(\mathrm{OR}=3.61$, $95 \% \mathrm{Cl}: 2.23-5.84)$ and serum prealbumin $(\mathrm{mg} / \mathrm{L})(\mathrm{OR}=2.88$, $95 \% \mathrm{Cl}: 1.04-8.00)$. However, they did not provide data on other inflammatory biomarkers in their article [17]. Liu G. found significant correlation of NRS-2002 with NRI ( $k=0.491$, 95\% Cl: 0.328-0.653; $p<0.001)$, and other anthropometric clinical measures (MNA-sf, and MUST; $p<0.001$ ) [12]. Del Giorno, et al. investigated the correlation of different anthropometric measures with loss of appetite and found significant correlation between NRS and loss of appetite ( $p$ $<0.001$ ) [9].

Two studies which used SGA showed significant association of clinical anthropometric measures with serum albumin $[2,8]$. Liu $\mathrm{H}$, et al. found significant association of inflammatory marker levels in malnourished group compared with normal group: peripheral blood lymphocyte count $(\mathrm{p}=$ $0.025)$; serum albumin $(p=0.019)$; serum IL-6 ( $p=0.016)$; peripheral blood $\mathrm{CD}^{+} \%(p=0.027)$. However, CRP level was not different among different groups $(P=0.259)$ [8] Similarly, Nicolau, et al. found significantly higher level of inflammatory markers in malnourished group compared with well-nourished patients [2].

The other two studies which used anthropometric measures could not show a significant association of inflammatory biomarkers with malnutrition $[18,19]$. Allard study divided the patients into two groups of malnourished (38.9\%) and without malnutrition (61.1\%) and did not find significant association between higher level of inflammatory markers of CRP, ferritin, fibrinogen, IL-6, LDH, and procalcitonin with malnutrition. However, they also regrouped the patients based on another index, named NRI into two groups of at nutritional risk and at no nutritional risk. The percentage of those who were grouped as at risk of malnutrition was $84 \%$, nutritional risk. Then significant association of CRP level ( $p$ $<0.01)$, fibrinogen $(p<0.01)$, and procalcitonin $(p<0.01)$ in at risk group compared with those who were not at risk of malnutrition were found [19].

In Bedock study BMI was associated with malnutrition ( $p<0.01$ ); lymphocyte count was significantly higher in malnourished patients compared with those who did not have malnutrition: $(1.45 \pm 0.62$ vs. $1.07 \pm 0.52)\left(\times 10^{9} / \mathrm{L}\right) ; p=$ $0.04)$. However, they did not find a significant association of CRP or D-dimer with malnutrition [18].

The last study in this group used MNA with an average MNA score of $22.9 \pm 2.8$ among patients. Malnourished group had significantly lower serum albumin $(25.7 \pm 5.3 \mathrm{~g} / \mathrm{L})$ compared to non-malnourished group $(38.5 \pm 4.2 \mathrm{~g} / \mathrm{L}, \mathrm{p}<$ $0.001)$. The similar significance was reported for peripheral blood lymphocyte count $\left(0.9 \pm 0.38\left(\times 10^{9} / \mathrm{L}\right)\right.$ in malnourished vs non-malnourished patients $(1.7 \pm 0.52, \mathrm{p}<0.001)$ [5].

\section{Studies which used laboratory markers to examine nutritional status}









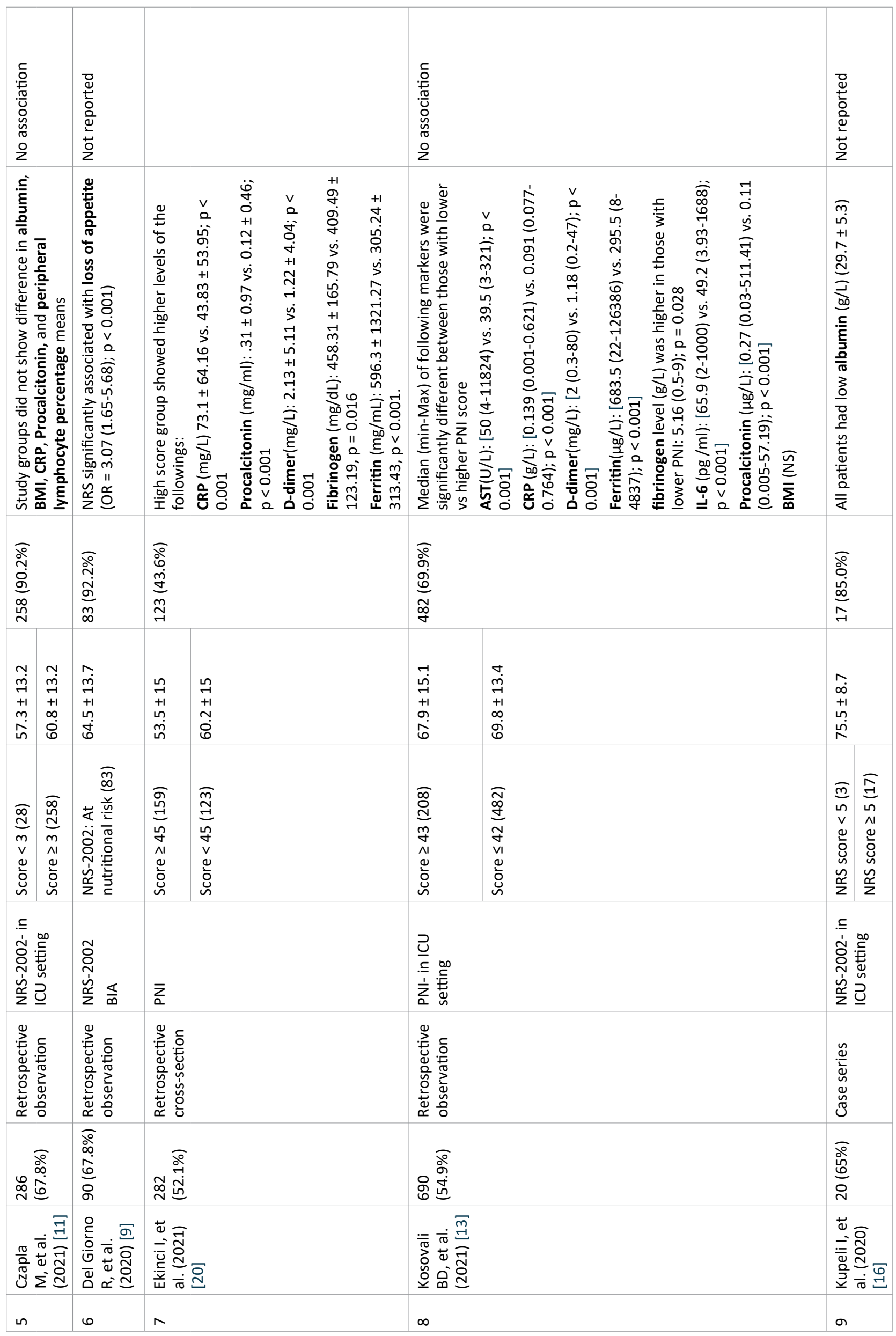




\begin{tabular}{|c|c|c|c|c|c|c|c|c|c|c|}
\hline \multicolumn{3}{|c|}{ 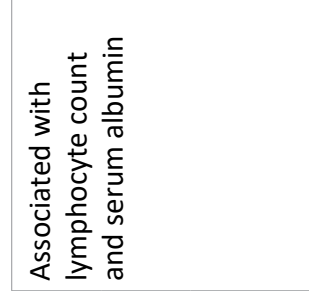 } & \multicolumn{2}{|c|}{ 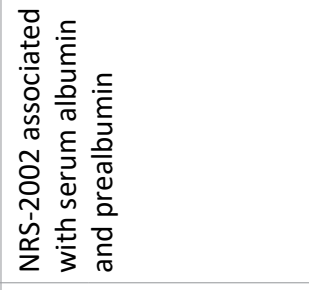 } & \multicolumn{2}{|c|}{ 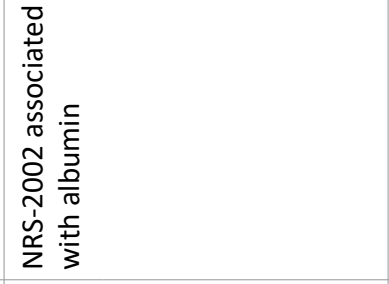 } & \multicolumn{4}{|c|}{ 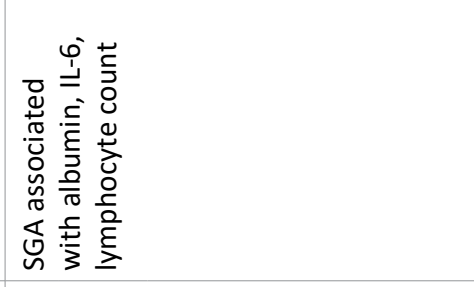 } \\
\hline \multicolumn{3}{|c|}{ 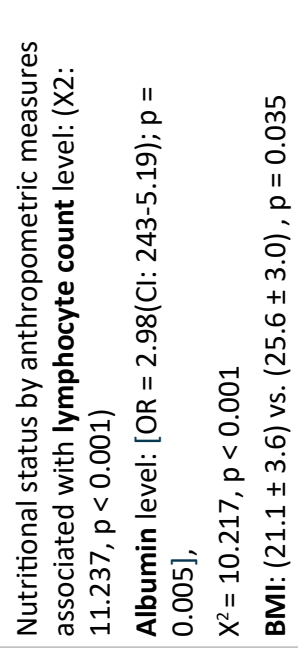 } & \multicolumn{2}{|c|}{ 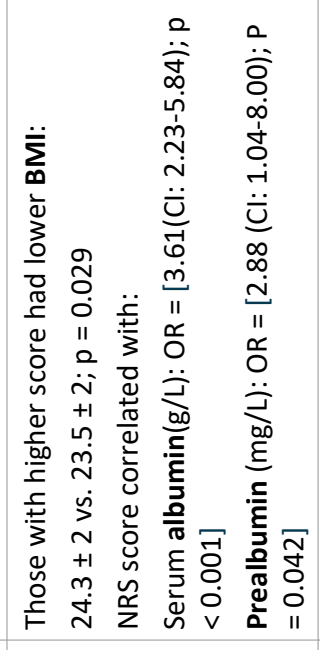 } & \multicolumn{2}{|c|}{ 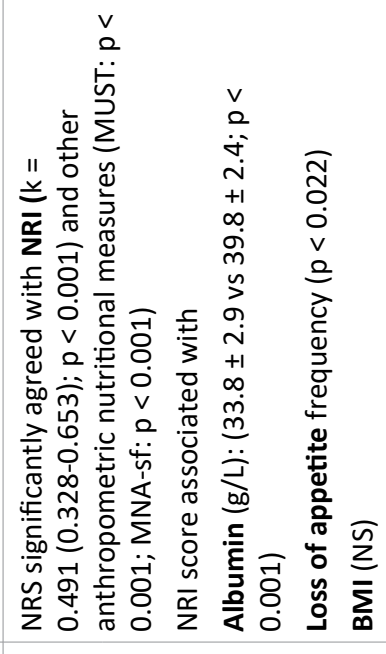 } & \multicolumn{4}{|c|}{ 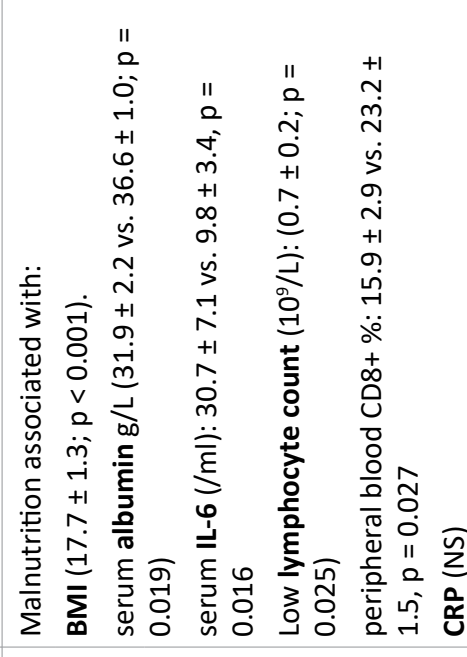 } \\
\hline \multicolumn{3}{|l|}{ 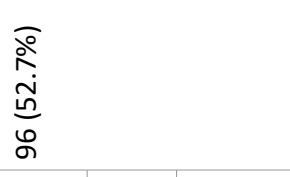 } & \multicolumn{2}{|l|}{$\begin{array}{l}\widehat{\alpha} \\
b \\
\dot{\infty} \\
\infty \\
\infty \\
\tilde{b}\end{array}$} & \multicolumn{2}{|l|}{ 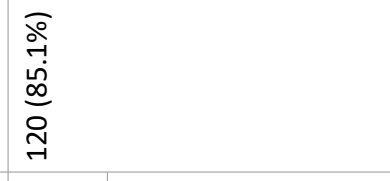 } & \multicolumn{4}{|l|}{$\begin{array}{l}\bar{\alpha} \\
\stackrel{\partial}{\overrightarrow{1}} \\
\infty\end{array}$} \\
\hline $\begin{array}{l}-1 \\
\infty \\
+1 \\
+1 \\
\infty \\
0\end{array}$ & $\begin{array}{l}m \\
\stackrel{m}{1} \\
+1 \\
1 \\
0 \\
0\end{array}$ & $\begin{array}{l}1 \\
\infty \\
\infty \\
+1 \\
\infty \\
0 \\
0\end{array}$ & $\begin{array}{l}\bar{p} \\
\infty \\
\infty \\
0 \\
g \\
g\end{array}$ & $\begin{array}{l}\overline{7} \\
0 \\
0 \\
0 \\
0 \\
0\end{array}$ & $\begin{array}{l}0 \\
\dot{+} \\
+1 \\
+1 \\
\stackrel{R}{R} \\
\end{array}$ & $\begin{array}{l}0 \\
\dot{0} \\
+1 \\
o \\
i \\
i\end{array}$ & $\begin{array}{l}a \\
i \\
+1 \\
+ \\
\dot{n} \\
0\end{array}$ & $\begin{array}{l}\overrightarrow{1} \\
\dot{+} \\
+1 \\
+ \\
\dot{1} \\
0\end{array}$ & $\begin{array}{l}9 \\
i \\
+1 \\
+1 \\
6 \\
6\end{array}$ & \\
\hline 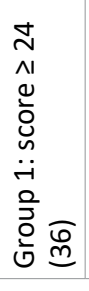 & 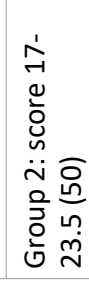 & 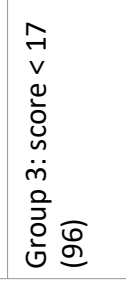 & 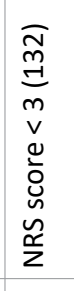 & 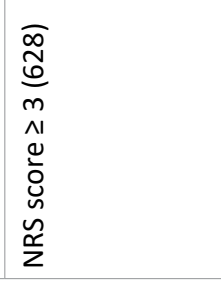 & 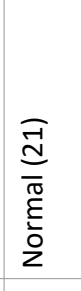 & 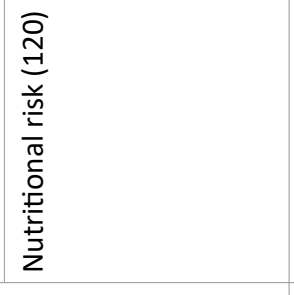 & & 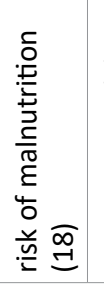 & 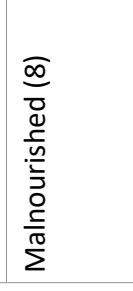 & \\
\hline \multicolumn{3}{|l|}{$\sum_{\Sigma}^{\nwarrow}$} & \multicolumn{2}{|l|}{ 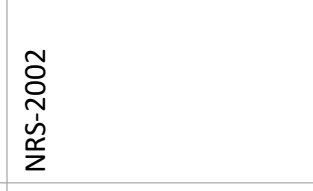 } & \multicolumn{2}{|c|}{ 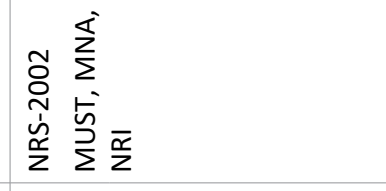 } & \multicolumn{4}{|l|}{ 忍 } \\
\hline \multicolumn{3}{|l|}{ 离 } & \multicolumn{2}{|c|}{ 竞 } & \multicolumn{2}{|c|}{ 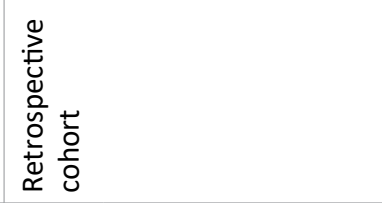 } & \multicolumn{4}{|c|}{ 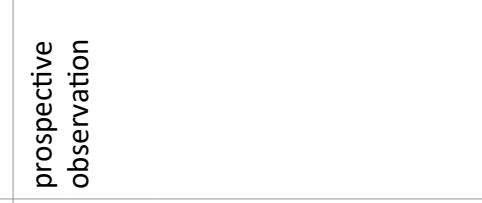 } \\
\hline \multicolumn{3}{|l|}{ 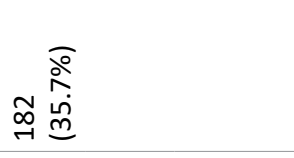 } & \multicolumn{2}{|l|}{$\begin{array}{l}\bar{o} \\
8 \\
0 \\
0 \\
0 \\
1\end{array}$} & \multicolumn{2}{|c|}{ } & \multicolumn{4}{|c|}{ 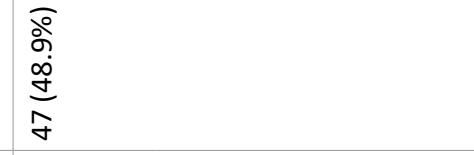 } \\
\hline \multicolumn{3}{|l|}{ 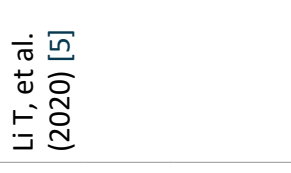 } & \multicolumn{2}{|c|}{ 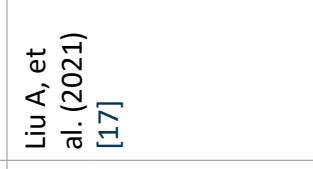 } & \multicolumn{2}{|c|}{ 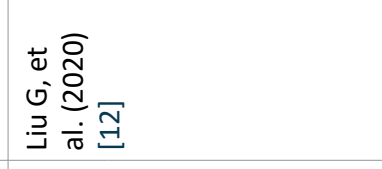 } & \multicolumn{4}{|c|}{ 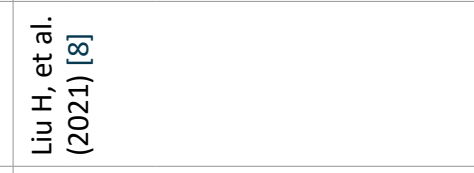 } \\
\hline \multicolumn{3}{|l|}{ 아 } & \multicolumn{2}{|l|}{$\exists$} & \multicolumn{2}{|l|}{$\approx$} & \multicolumn{4}{|l|}{$\stackrel{m}{\sim}$} \\
\hline
\end{tabular}




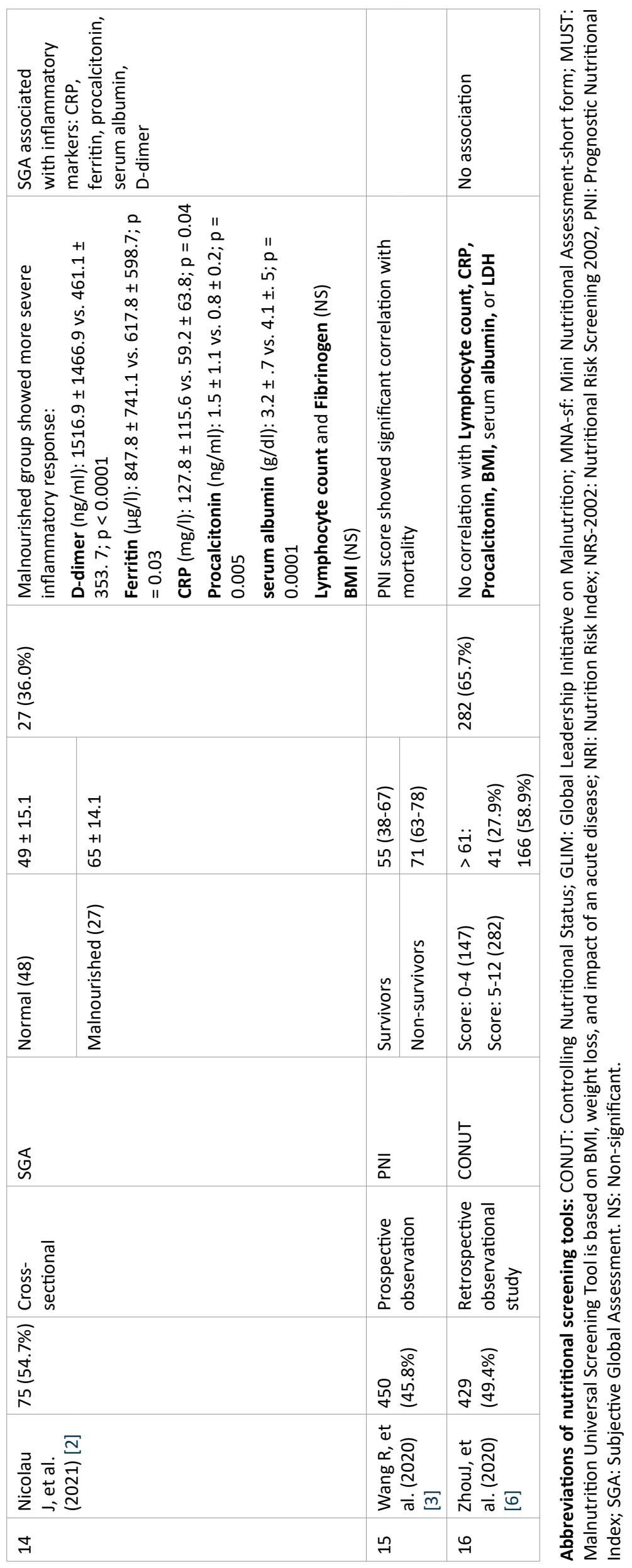


Citation: Sadighi S, Mohseni S, Hosseini SF, et al. (2022) The Association between Nutritional Parameters Used in Nutritional Risk Assessment (NRA) Tools in Hospitalized Malnourished COVID-19 Patients: A Systematic Review. J Healthcare 5(1):106-116

In one study, Cui, et al. used a serum prealbumin value of $150 \mathrm{mg} / \mathrm{L}$ as the cut-off level to define the malnourished group. They found $67.3 \%$ of the low prealbumin group had NRS score $\geq 4(p<0.001)$. Furthermore, the low prealbumin group showed a lower level of albumin, total cholesterol, and triglyceride; $p<0.001)$ and a higher level of inflammatory markers (Procalcitonin, CRP, IL-6; $p<0.001$ ) [21].

Three studies used PNI $[3,13,20]$. Kosovali, et al. studied patients admitted to the ICU and found significant association of inflammatory markers (lymphocyte count, serum albumin, D-dimer, CRP, and fibrinogen) with PNI $\leq 42$. However, BMI was not different between two groups of high and low PNI [13].

In Ekinci, et al. study, PNI mean in moderate or severe malnourished groups was $51.45 \pm 6.41$ versus $39.4 \pm 4.58$ in normal and mild malnourished groups. Of note, the levels of inflammatory markers in those with moderate to severe malnutrition in comparison with normal and mild malnourished groups were higher [20]. The average PNI in Wang, et al. study was $42.96 \pm 6.25$. They did not categorize the patients based on nutritional status. But they found significant association of PNI $(<0.001)$, AST $(<0.001)$, LDH $(<$ $0.001), \operatorname{CRP}(<0.001)$, and D-dimer $(<0.001)$ with survival [3].

In another study, CONUT score ranges from 0-12 and score 4 was used as the cut off score for dividing patients into highscore or low-score groups. The score did not correlate with BMI. Two groups did not show any difference in D-dimer level. Furthermore, the ratio of patients with high procalcitonin $(p=$ 0.089 ) or LDH level ( $p=0.651)$ was similar in two groups [6].

Regarding association of BMI with nutritional assessment tools, it should be mentioned that BMI, one of the main indices in defining nutritional status, was reported in seven studies which used clinical anthropometric measures $[2,5,8,11,12,17,18]$. Four out of those seven studies showed a significant association of BMI with nutritional state of patients $[5,8,17,18]$.

The three studies which used laboratory measures in defining the nutritional status of their patients and reported $\mathrm{BMI}$, did not find its significant association with nutritional status $[6,13,20]$. All studies which used anthropometric assessment tools found correlation of serum albumin with nutritional state except Four. Two of those four studies did not find any significant association $[13,18]$ and the other two studies did not report serum albumin of the patients $[9,19]$.

\section{Discussion}

To the best of our knowledge, this is the first systematic review intended to clarify the relationship between nutritional and inflammatory parameters in the mediation of malnutrition among COVID-19 patients. The most prominent result of the present study is the strong association of serum albumin as a nutritional biomarker with different NRA in COVID-19 patients.

Three studies which assessed nutritional status with NRS2002 in ICU and non- ICU patients, illustrated serum albumin is associated with malnutrition $[10,12,17]$. Researchers who used SGA as a screening tool similarly concluded that serum albumin is correlated with malnutrition $[2,8]$. This relationship means that serum albumin in the case of COVID-19 disease, despite the inflammatory circumstances, maintains its meaningful connection with the nutritional status of the patients. At the same time, inflammatory biomarkers including WBC, lymphocyte count, CRP did not reveal a correlation with most clinical-anthropometric tools. CRP and lymphocyte count had an association with PNI score that is basically laboratory and inflammatory based screening instrument.

The nutritional status in COVID-19 patients is a prognostic factor. Malnutrition can lead to adverse events through impaired immune defense mechanisms, reduced respiratory muscle mass and strength. The risk of ICU admission, mechanical ventilation, and mortality would be much higher in malnourished patients $[2,3]$.

As from the early stages of COVID-19 infection, most patients have fever and gastrointestinal symptoms such as loss of appetite, nausea, vomiting, and diarrhea, they can experience rapid and substantial weight loss $[10,11]$. On the other hand, there are some tools including, SGA, NRS2002, MNA and GLIM considering clinical anthropometric parameters such as body weight and BMI [4]. As a result, these tools alone could not be used as appropriate instruments for the diagnosis of malnutrition in hospitalized COVID-19 patients. Additionally, the lack correlation with MBI in Nicolau study was explained by the existence of recall bias by patients who did not recall their correct weight or the healthcare staff who could rely on their memory to document the results of clinical anthropometric measures [2] Finally, a significant association between malnutrition and BMI was reported only in four studies that used clinical-anthropometric nutritional tools $[5,8,17,18]$. Some patients with malnutrition had normal $\mathrm{BMI}$ or even high BMI. This discrepancy may be related to changing body composition due to fluid collection, especially in the ICU setting, underlying diseases, loss of muscle mass, and fat gain as a primary aging process or secondary to inflammatory condition (sarcopenia obesity). Recognition of malnutrition despite normal or increased BMI might lead to consideration of reduced muscle mass and sarcopenia's crucial role in nutritional assessment of COVID-19 patients.

Regarding inflammatory biomarkers, lymphocyte count was the most reported one. Lymphocyte count is the main factor considered in PNI tool, but did not show association with CONUT, another laboratory NRA tool.

Cui, et al. used prealbumin level as the nutritional screening measure in 408 patients and showed that lymphocyte count and NRS-2002 were associated with malnutrition based on pre-albumin level [21]. In three of four studies in ICU setting, nutritional status was examined with NRS-2002. Czapla, Kupli and Alikiaii did not find a significant correlation between NRS score and lymphocyte count as an inflammatory marker $[10,11,16]$. Although there is a clear consensus on albumin, no correlation is observed between lymphocyte count and malnutrition. Unexpectedly, one of the studies notified significantly higher lymphocyte count 
Citation: Sadighi S, Mohseni S, Hosseini SF, et al. (2022) The Association between Nutritional Parameters Used in Nutritional Risk Assessment (NRA) Tools in Hospitalized Malnourished COVID-19 Patients: A Systematic Review. J Healthcare 5(1):106-116

in severely malnourished patients compared to moderately malnourished and not malnourished patients $(p=0.04)$ [18]. Based on the results of this systematic review, lymphocyte counts as an indicator for malnutrition diagnosis might be an inappropriate test. Similarly other inflammatory parameters including CRP, LDH and WBC count did not show association with most of the NRA tools in this systematic review.

Using different tools may contribute to reaching different results. There is no gold standard tool for nutritional risk assessment among COVID-19 patients. Moreover, different studies used different cut-off points categorize patients in different nutritional groups. In those who used NRS-2002, Czapla, et al. and Liu A, et al. used score $3[11,17]$, Kupeli, et al. used score 5 [16], and Alikiaii, et al. separated patients into three groups of score 3, score 4, and more than score 5 [10]. In those who used PNI, Kosovali, et al. defined malnutrition by $\mathrm{PNI}<43$, [13] but Ekinki, et al. with comparable results used a higher cut off point of $<45$ in non-ICU patients [20]. Instead of using cut-off values, Wang, et al. introduced a prognostic model nomogram including PNI score, WBC, and LDH for predicting mortality of COVID patients [3].

The strength of this review is the inclusion of studies using different types of nutritional assessment tools which can be helpful in future studies to compare NRA. Eminently, data reported in the study was obtained from different research centers worldwide, covering ethnical and socioeconomic differences and leading to accurate information.

This study has some limitations. Since the information was only recorded from inpatient admission time without considering follow-up data, dynamic NRA and changing characteristics of nutritional status were not assessed. Secondly, even in an ICU setting, sarcopenia as one of the functional measures to assess nutritional status in the elderly was not covered in included studies. Finally, Coronavirus strains that can significantly alter patients' symptoms have not been determined in these studies.

\section{Conclusion}

There are no uniform or gold standard nutritional screening and assessment tools for COVID-19 patients. This review shows that selected articles used various nutritional screening tools to assess the effect of inflammatory biomarkers and nutritional measures in diagnosing malnutrition. However, there remains a lack of consensus on which measurement is accurate or appropriate for determining nutritional risk in hospitalized COVID-19 patients. COVID-19 infection influences nutritional parameters and inflammatory biomarkers, including albumin, lymphocyte, and WBC count; consequently, such parameters used in nutritional screening tools might most likely be influenced by the natural course of the infection.

Selecting the most appropriate method to assess the nutritional status of COVID-19 patients should be chosen based on resources and level of risk in the population. For rapid malnutrition screening in ambulatory patients, simple, low-cost risk assessment tools that included nutritional and anthropometric measures like nutritional risk index could be practical. However, accurate malnutrition diagnosis in a hospital setting, to eliminate the undesirable complications, necessitates a comprehensive nutritional assessment considering muscle mass and function would be more reliable for prevention measures, nutritional treatment decisions and dietary modifications.

\section{References}

1. Simonnet A, Chetboun M, Poissy J, et al. (2020) High prevalence of obesity in severe acute respiratory syndrome Coronavirus-2 (SARS-CoV-2) requiring invasive mechanical ventilation. Obesity (Silver Spring) 28: 1195-1199.

2. Nicolau J, Ayala L, Sanchís P, et al. (2021) Influence of nutritional status on clinical outcomes among hospitalized patients with COVID-19. Clin Nutr ESPEN 43: 223-229.

3. Wang R, He M, Yin W, et al. (2020) The prognostic nutritional index is associated with mortality of COVID-19 patients in Wuhan, China. J Clin Lab Anal 34: e23566.

4. Cederholm T, Jensen GL, Correia MITD, et al. (2019) GLIM criteria for the diagnosis of malnutrition-A consensus report from the global clinical nutrition community. J Cachexia Sarcopenia Muscle 10: 207-217.

5. Li T, Zhang Y, Gong C, et al. (2020) Prevalence of malnutrition and analysis of related factors in elderly patients with COVID-19 in Wuhan, China. Eur J Clin Nutr 74: 871-875.

6. Zhou J, Ma Y, Liu Y, et al. (2021) A correlation analysis between the nutritional status and prognosis of COVID-19 patients. J Nutr Health Aging 25: 84-93.

7. Jia $\mathrm{H}$ (2016) Pulmonary angiotensin-converting enzyme 2 (ACE2) and inflammatory lung disease. Shock 46: 239-248.

8. Liu H, Zhou L, Wang $\mathrm{H}$, et al. (2021) Malnutrition is associated with hyperinflammation and immunosuppression in COVID-19 patients: A prospective observational study. Nutr Clin Pract 36: 863-871.

9. Del Giorno R, Quarenghi M, Stefanelli K, et al. (2020) Nutritional risk screening and body composition in COVID-19 patients hospitalized in an internal medicine ward. Int J Gen Med 13: 1643-1651.

10. Alikiaii B, Heidari Z, Fazeli A, et al. (2021) Evaluation of the effectiveness of the nutritional risk screening system 2002 (NRS2002) in COVID-19 patients admitted to the intensive care unit. Int J Clin Pract 75: e14934.

11. Czapla M, Vela RJ, Caballero VG, et al. (2021) The association between nutritional status and in-hospital mortality of covid-19 in critically-ill patients in the ICU. Nutrients 13: 3302.

12. Liu G, Zhang S, Mao Z, et al. (2020) Clinical significance of nutritional risk screening for older adult patients with COVID-19. Eur J Clin Nutr 74: 876-883.

13. Kosovali BD, Kucuk B, Soyal OB, et al. (2021) Can prognostic nutritional index predict mortality in intensive care patients with COVID-19? Int J Clin Pract 75: e14800.

14. Li W, Li M, Wang T, et al. (2020) Controlling Nutritional Status (CONUT) score is a prognostic factor in patients with resected breast cancer. Sci Rep 10: 6633.

15. Wells G, Shea B, O'Connell D, et al. (2021) The newcastle-ottawa scale (NOS) for assessing the quality of nonrandomised studies in meta-analyses. 
Citation: Sadighi S, Mohseni S, Hosseini SF, et al. (2022) The Association between Nutritional Parameters Used in Nutritional Risk Assessment (NRA) Tools in Hospitalized Malnourished COVID-19 Patients: A Systematic Review. J Healthcare 5(1):106-116

16. Kupeli I, Yuksel F (2020) Coronavirus (COVID-19), advanced age, and malnutrition: A risky coexistence. Aging Medicine and Healthcare 11: 142-145.

17. Liu A, Cong J, Wang Q, et al. (2021) Risk of malnutrition is common in patients with coronavirus disease 2019 (COVID-19) in Wuhan, China: A cross-sectional study. J Nutr 151: 1591-1596.

18. Bedock D, Lassen PB, Mathian A, et al. (2020) Prevalence and severity of malnutrition in hospitalized COVID-19 patients. Clin Nutr ESPEN 40: 214-219.

19. Allard L, Ouedraogo E, Molleville J, et al. (2020) Malnutrition: Percentage and association with prognosis in patients hospitalized for coronavirus disease 2019. Nutrients 12: 3679.

20. Ekinci I, Uzun H, Utku IK, et al. (2021) Prognostic nutritional index as indicator of immune nutritional status of patients with COVID-19. Int J Vitam Nutr Res 92: 4-12.

21. Cui $\mathrm{N}$, Tong $\mathrm{H}$, Li Y, et al. (2021) Role of prealbumin in predicting the prognosis of severely and critically ill COVID-19 patients. Am J Trop Med Hyg 105: 718-726.

DOI: $10.36959 / 569 / 471$

Copyright: (c) 2022 Sadighi S. et al This is an open-access article distributed under the terms of the Creative Commons Attribution License, which permits unrestricted use, distribution, and reproduction in any medium, provided the original author and source are credited. 